\title{
Putting Mass Spectrometry in the Hands of the End User
}

\author{
Frank S. Pullen, George L. Perkins, and Keith I. Burton \\ Pfizer Ltd., Ramsgate Road, Sandwich, Kent CT13 9NJ, England \\ Richard S. Ware, Mathew S. Teague, and Jeffrey P. Kiplinger \\ Pfizer Inc., Eastern Point Road, Groton CT 06340 USA
}

\begin{abstract}
The ease of use of the newer liquid chromatography-mass spectrometry interfaces has made possible the automated acquisition of spectra from large batch queues of samples. This fact, combined with the realization that unit molecular mass determination was the only datum desired by a majority of drug discovery synthetic chemists, led us to develop open access mass spectrometry in the early 1990s. Open access spectrometers now scan over 100,000 samples per year from synthesis laboratories at Pfizer. Our experiences with this novel use of mass spectrometry in a large research facility are discussed and we detail some of the pitfalls we believe to be common to this approach. In addition, we offer some reflection on the cultural changes we have observed in our research environment since this experiment began. (J Am Soc Mass Spectrom 1995, 6, 394-399)
\end{abstract}

$\mathrm{T}$ The last several years have seen an increasing interest in automation of routine mass spectrometry. The addition of autosamplers to gas chromatography-mass spectrometry (GC/MS) systems was of benefit to the many laboratories that need to run large batches of samples under standard conditions [1]. In the late 1980s, techniques for automation often were applied in cases where the mass spectrometer source and interface parameters could be tuned to set conditions for a particular repeated analysis, as in environmental [2] or drug metabolism [3] selected-ion monitoring applications. Automated probe designs began to surface in the late 1980s [4]; they offered a method whereby complete electron ionization (EI) or chemical ionization (CI) spectra might be acquired on large batches of chemically distinct samples. More recently, the application of thermospray (TSP) interfaces to the analysis of batches of samples introduced through these liquid chromatography (LC) interfaces [5] has significantly extended the range of chemical classes amenable to analysis. Ionization methods such as electrospray ionization (ESI), particle beam (PB), and TSP, which are reliable means of direct introduction of samples to the mass spectrometer from solution and are applicable to a wide variety of chemical structures, offer new possibilities in automated spectrometry [6]. With the increased stability and ease of use of microprocessor-

Address reprint requests to Jeffrey $P$. Kiplinger, Pfizer Central Research, Eastern Point Road, Groton, CT 06340. controlled instruments, robust ion optics and detectors, and liquid chromatography-mass spectrometry (LC/ MS) interfaces, more laboratories use autosamplers with solvent delivery systems to deliver samples in solution to ionization sources. These technological advances brought automated mass spectrometry to the attention of the pharmaceutical industry, which generates huge numbers of unique chemicals as part of drug discovery programs.

To support such programs, one of our most important routine tasks is to confirm structure quickly. Although the term is distasteful to many spectroscopists, this is a "service job" that requires little intellectual effort but much time. Chemists at Pfizer routinely gather data to support the structure assignments of synthetic products prior to drug screening, primarily through use of open access, or "self-service," NMR systems. Molecular mass measurement is the most easily interpreted structure confirmation technique available to the chemist; a single number either supports or refutes putative structure. Although it does not actually confirm structure, the chemist is able to observe that a predicted mass change has taken place from starting material to product, and this provides enough confirmation of a successful synthesis to proceed to the next synthetic step. When our laboratory acquired the ability to routinely acquire fast-atom bombardment (FAB) spectra in the mid-1980s, chemists quickly realized that this technique offered them mass measurement for virtually all their polar and labile 
synthetic targets. By 1989, we were inundated with requests for fast-turnaround $\mathrm{FAB}$ spectra (or EI, $\mathrm{CI}$, etc., when appropriate), which were of interest only to the chemists as a measurement of monoisotopic nominal mass.

The realization that the chemists rarely required any mass spectral interpretation initiated a search for a new paradigm. We began to consider ways in which the spectroscopist's time outlay to generate routine spectra could be minimized so that more time was available for problem-solving applications. The result was that by 1992, seven mass spectrometers were available to Pfizer chemists in Sandwich (UK) and Groton (US) as fully automated and open access systems. At present, instead of submitting samples to a mass spectrometry laboratory for analysis, chemists independently use these instruments in self-service instrument laboratories. They prepare samples in solvent to a suggested range of concentration in an autosampler vial, log the sample into the data system, and come back later to examine the data. On some of the instruments, the spectrum is automatically printed. The chemists interact with spectrometrists only if necessary.

A critical distinction should be made here between open access mass spectrometry and automated molecular mass confirmation. The former requires a new instrument design, which allows chemists to add new samples to the autosampler at any time during acquisition, new fail-safe designs to prevent problems due to continuous interaction with untrained users, and new start-up and shut-down systems that allow 24-h operation. The latter is well accommodated by existing software and spectrometers with autosamplers, and requires only that a trained operator load a batch queue of samples that require identical analyses. Automated mass spectrometry of batches of samples is not the same as an open access system with slightly more control exercised by a mass spectrometrist. We have, over a two-year period, refined and added to the designs of our various open access spectrometers to the point that one of them is now marketed as a separate product line by an instrument vendor (Fisons OPEN ACCESS, Fisons Instruments Inc., Danvers, MA). The two approaches require and instill a different attitude about mass spectrometry, both among spectrometrists and among chemists that require our services. As an example, an ASMS workshop on automated mass spectrometry techniques involved comparison of our work with that of Carr and Bean (SmithKline Beecham) on automated electrospray and Matuszak (Abbot) on robot desorption chemical ionization probe analysis of batches of pharmaceutical samples for molecular mass confirmation [6]. The controversy that ensued over the wisdom of putting mass spectra directly into the hands of the end user illustrates the significant difference between the approaches.

We chose not to develop automated methodology for processing batches of samples within the mass spectrometry laboratory because this alternative did not offer the advantages we most desired: fast return of spectra to the chemists and relief for spectrometrists from the mundane task of sample handling.

The most significant result of this new approach at Pfizer is that a mass spectrum is now easier to obtain than any other single piece of analytical data. Many chemists now use mass spectrometry in the same way that they formerly used thin layer chromatography (TLC)- to routinely check a reaction for the presence of product. Our open access spectrometers currently provide data on over 120,000 samples per year. We predict that this number will increase to close to 250,000 in 1995.

As previously mentioned, the prevailing sentiment among attendees at the 1993 ASMS workshop was that providing chemists with spectra without interpretation is, at best, risky. Misinterpretation of data by nonexperts might lead chemists to draw incorrect conclusions about structure, and the mass spectrometry laboratory would share liability for such mistakes. Our experiences with open access mass spectrometry show that this is indeed one of the risks in this venture, and it needs to be addressed. However, the astounding success of our move to give our end users (synthetic chemists) their own mass spectrometers shows that this risk is worth accepting.

Within the last year, reports have begun to appear in the literature that detail automated mass spectrometry methods for the analysis of small polar molecules [5]. A recent report at this year's ASMS meeting [7] indicated that other pharmaceutical companies are experimenting with open access mass spectrometry. This account is our effort to share our early experiences. This novel way to use mass spectrometry was implemented at Pfizer concurrently in both US and UK research facilities, and most of the problems and pitfalls were encountered at both research sites. This indicated to us that these problems probably were not unique and that our experiences might be useful to others.

\section{Experimental}

It is not the intention of this letter to describe in detail the configuration and operation of the open access spectrometers at Pfizer. The GC/MS systems [two Hewlett Packard (Avondale, PA) HP-5971A gas chromatography mass spectrometry detectors (GC/MSDs) at Groton and one Fisons Instruments MD-800 at Sandwich] are standard configurations as marketed by their manufacturers, with no customization. Their operating parameters and conditions are normal and routine for 12-m low polarity capillary columns, and a fast temperature ramp is used to ensure that the column offers some limited separation based on boiling point. The Fisons thermospray-mass spectrometry (TSP/MS) system, based on a Trio-1000 spectrometer (Fisons), has 
been described extensively elsewhere [8]. A Fisons Trio-1000 in Groton is configured similarly. The second Fisons Trio-1000 system at Sandwich and the HP-5989 system at Groton are configured with the vendors' standard particle beam (PB) interface and with ammonia $\mathrm{CI}$ ionization, but the customized software is designed so that each instrument functions in a manner similar to the prototype Fisons TSP/MS system. At both sites, the particle beam spectrometers are down about 2 days per month, whereas the Trio-1000/TSP system has proven somewhat more sturdy, with about 2 hours of downtime per month.

Briefly, three basic modifications were necessary for both the Hewlett-Packard and Fisons TSP/MS and $\mathrm{PB} / \mathrm{MS}$ spectrometers. First, a sample log-in routine was added to the data system - on a separate computer for the Fisons instruments and as the start-up window of a turnkey system on the Hewlett-Packard. Second, both spectrometers required modification of the data acquisition routines to allow samples to be added to the queue at any time, whether during acquisition or not. Finally, both systems were set up to revert to an "idle" state when some time had passed without activity; this state shuts down the LC pumps to remove solvent load from the vacuum pumps and cools the thermospray jet. As part of the modification, both types of instruments are configured to automatically return to an active condition when a chemist logs in a new sample. A period of debugging and some amount of reworking of this new software was necessary following installation of the prototype instruments, but these added features work smoothly at present.

\section{Experiences with the Hardware}

Our initial experiment with opening instrumentation to synthetic laboratories was tentative: we used proven technology that did not require extensive training or development effort. A Hewlett-Packard GC/MSD system was installed in the mass spectrometry laboratory (not the self-service laboratory) for three months in early 1990 to determine whether sufficient interest in self-service mass spectrometry existed. Drug discovery chemists were invited to bring samples and run them with the help and coaching of an operator. This break-in period allowed us to fine tune the operating parameters of the spectrometer and gas chromatograph to be most useful for the widest variety of samples; to develop a standard experiment time, temperature ramp, and data output format; and to determine which classes of compounds were unsuited to this type of analysis due to involatility or ionization problems. At the end of this period, the spectrometer was moved to a selfservice instrument laboratory and several formal 20min training sessions were offered to users. The instrument has been fully operational greater than $95 \%$ of the time since installation. We acquired a second GC/MS instrument six months later, and an MD-800 from Fisons instruments was added in Sandwich to serve the UK discovery chemists.

The GC/MSD systems were limited with respect to higher mass and less volatile samples. We needed a benchtop spectrometer with a higher mass range and an LC/MS interface so that samples could be introduced directly in solution. In 1991, mass spectrometry laboratory personnel at Sandwich began to evaluate the different available $L C$ interfaces to determine which one provided molecular mass information on the greatest variety of synthetic compounds from Pfizer discovery laboratories. Although it was determined through extensive testing that no single interface would handle all the sample types likely to be encountered (Sugden, J.; Pullen, F. S.; Bowen, D. V., manuscript in preparation) thermospray was found useful for $80-90 \%$ of the samples commonly submitted for mass measurement. Fisons Instruments agreed to customize a Trio-1000 benchtop spectrometer for this application, and this instrument has been described in detail elsewhere [8]. Groton spectrometrists were prepared to order a similar instrument, but first tested the particle beam interface with ammonia $C I$ on the HewlettPackard HP-5989 mass spectrometry engine. This test of compounds synthesized in discovery laboratories was as successful as the thermospray test, and Hewlett-Packard was chosen as the vendor of the US-based system. Both of these instruments were installed in 1992-the Hewlett-Packard system six months later than the Fisons system.

It is not our intent to present a survey of which compounds of interest to Pfizer's discovery chemists are best ionized by TSP, PB/CI mass spectrometry, or any other technique. We have noted several series of compounds that appear better suited to one technique than the other; for example, certain tetracyclines produce $\mathrm{MH}^{+}$ions by TSP but not by $\mathrm{PB} / \mathrm{CI}$ mass spectrometry. In practice, discovery chemists simply want a molecular mass and are not at all interested in how it is obtained. Our user training suggests that GC/EI mass spectrometry be used for samples of lower polarity and molecular mass, because the mass range of the MSDs is limited and the sample must pass through the GC column without derivatization. We also suggest that volatile samples not be analyzed by particle beam. However, it is up to the chemist to choose the spectrometer, and we sometimes find that the choice is arbitrary rather than carefully considered. Proximity of the instrument is a big factor, so that in Groton, where the instruments are located in different areas of the research complex, TSP is preferred by chemists located close to the TSP instrument and PB is preferred by others. A chemist may, in addition, have a "favorite" technique or instrument, which determines the choice. Our strategy has been to choose an ionization method or methods that allow molecular mass determination of the largest number of samples generated by drug discovery chemists. Although TSP, PB/CI mass spectrometry, atmospheric pressure $\mathrm{CI}$, and ESI seem the 
most suited to molecular mass determination among polar pharmaceutical molecules, or GC/EI mass spectrometry seems to handle the very small molecules that are not amenable to these methods. Another industry or application might wish to configure an open access system differently.

Both liquid introduction mass spectrometry systems interact with users in the same way that the GC/MSD systems do-samples go into an autosampler tray and users log samples into a running batch queue through a PC-based data system that runs a log-in program. Samples are loop-injected into the solvent stream delivered to the interface. Because no chromatography is available, data analysis is noninteractive (the user is not required to identify the total ion current peak of interest) and automatic, and the spectrum is automatically printed. At first glance, the instruments are simpler than the GC/MSD systems, which require the user to be familiar with the interactive data analysis software. In practice, these spectrometers offer higher mass ranges and more options than the MSD detectors, and are therefore more problem-prone. Most of the problems we have experienced are because of our attempts to use these instruments in ways the manufacturer did not anticipate, as the following example points out.

The autosampler itself is the source of a variety of problems. On all the instruments, regardless of manufacturer and type of interface, we use Hewlett-Packard autosamplers. This offers a substantial advantage because it is necessary to stock only one type of autosampler vial and snap-cap. The sampling arm cannot be touched when in operation (a substantial fraction of the 3-min sampling cycle on the TSP and PB instruments). However, users occasionally do contact the arm when loading new samples, which generates a fatal error that suspends operation of the system because the autosampler can no longer locate the next sample. It is not possible, due to the design of the communication link between the LC and the spectrometer, to ignore the error and reset the arm without disabling the communication link, thus losing the data system control of the LC and autosampler. Because the GC-based spectrometers operate on a 14-min sampling cycle, this is an infrequent problem. This type of problem is derived from the vendor's idea of the "intended use" of the instrument; in this case, repeated interactive modification of the batch queue was not planned for in the design of the autosampler. For both the Fisons and the Hewlett-Packard software, custom programming was added by the vendor to allow samples to be added to the queue during data acquisition. However, neither company has successfully circumvented the firmware-generated autosampler fault that results from contact with the arm.

Both Fisons and Hewlett-Packard have offered extensive software support in an effort to make their instruments function in this demanding environment. Frustration on both sides can run high. Almost invari- ably, recurrent hardware problems that are a surprise to both vendor and customer result from the use of a mass spectrometer in a way no one ever imagined or intended. The phrase "No one has ever broken one of those before" is frequently heard from our vendor representatives.

As more vendors offer benchtop spectrometers that are adaptable to an open access environment, anticipation of the potential pitfalls will become part of the design process. The Hewlett-Packard autosampler might better be designed for open access use if, when bumped, it simply skips the current sample, recenters its arm, generates an error message, and goes on to the next vial. We do not wish to recommend an individual system based on our experience at the present time; rather, we wish to urge potential purchasers of open access systems to work closely with any chosen vendor to design a workable instrument for the enyironment. Eventually, competition between vendors will encourage the development of instruments designed for this application.

\section{Experiences with Users}

The biggest difficulty with open access mass spectrometry has been the strong user dependence on the instruments. Confirmation of the appearance of a product in a synthesis was previously accomplished by TLC, followed by further workup and NMR or IR. Quick molecular mass measurement, when it became available through the open access machines, immediately became the preferred structure confirmation technique. This led to the following problems, many of which we did not anticipate simply because we did not anticipate the success of this venture.

First, and most important, downtime must be kept to a minimum. In fact, these spectrometers cannot go down without a direct impact on medicinal chemistry laboratories. Any routine maintenance must be carried out during low-use periods so that synthetic laboratory programs are disrupted as little as possible. Any unscheduled downtime is a critical situation that demands immediate action by mass spectrometry laboratory personnel. The message gleaned from our first year of operation was that a single instrument of any type is not enough; at least one backup must be available. In addition, at both research facilities it was necessary to install a full time technical support person who has responsibility for keeping the instruments operational.

Second, it is a natural tendency among synthetic chemists to carry out projects as quickly as possible. This contributes to a number of "inappropriate uses" of the spectrometers. For example, aliquots of reaction mixtures are sometimes injected into the PB or TSP mass spectrometers, which offer no chromatography and are protected only by an in-line filter for particulate removal. At best, the chemist may see a weak 
$\mathrm{MH}^{+}$ion (due to chemical interferences) from the desired product; at worst, the instrument may be contaminated with undesirable materials (polymerized reactants, salts) that reduce instrument sensitivity and the time interval between cleanings. The best solution to this type of problem is to require more extensive cleanup of samples, but this requires additional time from the synthetic chemist. Likewise, a weak or nonexistent protonated product molecule may encourage chemists to overconcentrate samples, which contributes to source contamination. The weak $\mathrm{MH}^{+}$ion may be due to a molecule that is involatile or poorly ionized by the interface. Although the best solution is to carry out the analysis by different techniques, this is often more time-consuming.

We have found that some education is necessary beyond teaching users how to load samples and obtain data. A minimum of 90 minutes of instruction is required at Groton for new users of the open access spectrometers. Much of this focuses on the way the instruments work and how they may be damaged, and on ionization processes and simple spectral interpretation skills. This foundation allows synthetic organic chemists to take partial responsibility to limit potential damage of samples and misuses of the spectrometers, and has reduced the incidence of problems of this type.

Finally, we have experienced more problems with misinterpretation of data than initially expected. Unsophisticated users of mass spectrometry are much less critical of the spectra they obtain than trained spectrometrists, and again some level of education is necessary to prevent problems. The critical concepts that must be made clear to users are (1) signal-to-background, which includes recognition that some background is normal, (2) peaks due to stable isotopes, noting especially that a peak without a carbon isotope peak is probably artifactual, and including recognition of common halogen isotope patterns, and (3) nitrogen rules. With this level of understanding, users are equipped to differentiate useful spectra from those that contain no information, and can determine whether an observed $\mathrm{MH}^{+}$or $\left[\mathrm{M}+\mathrm{NH}_{4}\right]^{+}$ion is likely to be derived from their desired product.

In addition, we were surprised by some unanticipated uses of these instruments that chemists discovered on their own. For example, the GC/MS instruments offer chromatographic separation of mixtures, but we had thought that the column would be most useful as a device for protecting the mass spectrometry from involatile compounds. Early on, chemists began to use the GC/MS instruments as reaction monitoring devices to profile the appearance of the product over time or varying conditions to optimize reactions. Again, mass spectrometry is replacing TLC in synthetic laboratories! The related problem we have had to correct is a tendency to use relative chromatographic peak areas to measure reaction yields.

\section{Summary}

In 1989, none of us would have predicted the astonishing success of our experiment with open access mass spectrometry. This year, a staff of two will oversee seven open access instruments, which will provide structure confirmation on over 120,000 newly synthesized compounds. By contrast, our remaining staff of seven supervisors and spectrometrists will use five spectrometers to generate about 10,000 spectra. Fortunately, our expertise is still needed; otherwise we might well have made ourselves obsolete. The need for fast molecular mass measurement among discovery chemists in the pharmaceutical industry is now easily recognizable, and new mass spectrometry technology is being applied to meet that need. Most impressive has been the cultural change that has taken place at Pfizer: mass spectrometry is now the primary structure confirmation tool; it even replaces TLC for product detection in some cases.

A question frequently raised in discussions with spectroscopists in the industry is whether the implementation of open access mass spectrometry creates unnecessary demand. Are 120,000 spectra per year more than is necessary for the number of chemists supported? The answer is undoubtedly yes. However, the fact that more spectra than necessary are being generated simply means that the chemists, who are in control of this technology, may wish to more carefully consider whether this measurement is necessary for a particular sample. It does not, in our opinion, imply that we should reduce access to the data or this technology. To do so would place limits on chemists who use open access mass spectrometry to increase their efficiency and productivity, and thus to generate new pharmaceuticals.

In spite of the substantial headaches involved in bringing these spectrometers on line, we are very pleased with the strong user dependence on the data. It is this dependence, however, that creates the headaches, because there is considerable pressure on the mass spectrometry laboratory to keep all open access spectrometers functioning at all times. Some of this pressure has been alleviated through education, through which users learn that they share responsibility to keep these instruments running well. User education also has minimized problems with misinterpretation, although these problems have proven to be less prevalent than we had feared. A reviewer of this paper raised an important question about the risks of misinterpretation by users: "Do mass spectrometrists share in the risks, or [are they] the chemists' responsibility?" It is our feeling that we do indeed share in the risks, because Pfizer expects our expertise to further the science of drug discovery and development. This is why we feel that proper user education is a critical responsibility in providing self-service mass spectrometry. 
An unavoidable conclusion from our experiences is that it will be impossible for other pharmaceutical firms or any other chemical industry that requires frequent confirmation of synthetic structures to function efficiently in the future without opening mass spectrometers directly to the end-users. The need for fast structure confirmation in a competitive industry is far too great and the technology is available to meet it. This is not too bold a statement, given the pressures common to drug discovery chemists within the industry. As chemists move from company to company, those that have experience with open access mass spectrometry will demand it in their new positions. If a technology exists that allows chemists to confirm molecular structure to their satisfaction within minutes, a competitive company cannot afford to ignore it.

\section{References}

1. (a) Brunee, C. Int. J. Mass Spectrom. Ion Phys. 1982, 45, 51; (b) King, G. S. Anal Proc. (London) 1982, 19, 261.

2. (a) Gilbert, J.; Startin, J. R.; Crews, C. Food Addit. Contam. 1985,
2, 55; (b) Jennison, C. P. R.; Jennison, J. Can. Chem. News 1991, 43, 23; (c) Vickers, A. K.; Wright, L. M. J. Automat. Chem. 1993, 15, 133; (d) Helmig, D.; Greenberg, J. P. I. Chromatogr. A 1994, $677,123$.

3. (a) Lant, M. S.; Oxford, J.; Martin, L. E. J. Chromatogr. 1987, 394, 223; (b) Foerster, H. J.; Rominger, K. L.; Ecker, E. M.; Peil, H.; Wittrock, A. Methodol. Surv. Biochem. Anal. 1992, 22, 231; (c) Matsushima, N.; Kwabata, K.; Natanai, T.; Sasahara, K. Nippon Iyo Masu Supekutoru Gakai Koenshu 1993, 18, 169; (d) Schmid, J.; Buecheler, A.; Mueller, B. J. Chromatogr. B 1994, $658,93$.

4. (a) Kath, G. S.; McKeel, W. J.; Smith, J. L.; Liesch, J. M. Rev. Sci. Instrum. 1986, 57, 3114; (b) Martin, D. J.; Bond, P. M. Biomed. Environ. Mass Spectrom. 1989, 18, 733; (c) Lee, M. J.; Organ, A. J.; Sims, J. L. Lab. Rob. Automat. 1993, 5, 149.

5. (a) Hayward, M. J.; Snodgrass, J. T.; Thomson, M. L. Rapid Commun. Mass Spectrom. 1993, 7, 85; (b) Tiller, P. R.; Lane, S. J. Rapid Commun. Mass Spectrom. 1993, 7, 1055.

6. Laboratory Managers Interest Group, Proceedings of the 41st ASMS Conference on Mass Spectrometry and Allied Topics; San Francisco, CA, 1993; p 1104.

7. Taylor, L. C. E. Proceedings of the 42nd ASMS Conference on Mass Spectrometry and Allied Topics; Chicago, IL, 1994; 322.

8. Brown, D. V.; Dalton, M.; Pullen, F. S.; Perkins, G. L.; Richards, D. Rapid Commun Mass Spectrom. 1994, 8, 632. 